Article

\title{
Optimization of Nonspherical Gold Nanoparticles for Photothermal Therapy
}

\author{
Paerhatijiang Tuersun ${ }^{1,2}$, Xiayiding Yakupu ${ }^{2}$, Xiang'e Han ${ }^{3, *(1)}$ and Yingzeng Yin ${ }^{1, *}$ \\ 1 National Key Laboratory of Antennas and Microwave Technology, School of Electronic Engineering, \\ Xidian University, Xi'an 710071, China; ptuersun@163.com \\ 2 Laboratory of Novel Light Source and Micro/Nano-Optics, School of Physics and Electronic Engineering, \\ Xinjiang Normal University, Urumqi 830054, China; xyakupu@163.com \\ 3 School of Physics and Optoelectronic Engineering, Xidian University, Xi'an 710071, China \\ * Correspondence: xehan@mail.xidian.edu.cn (X.H.); yzyin@mail.xidian.edu.cn (Y.Y.); \\ Tel.: +86-029-8820-2673 (X.H.)
}

Received: 3 September 2019; Accepted: 8 October 2019; Published: 13 October 2019

Featured Application: The optimization approach presented in this study can be directly applied to other metals by changing the parameters in the size-dependent refractive index. Complex shaped nanoparticles can also be optimized by using the discrete dipole approximation and the finite-difference time-domain method.

\begin{abstract}
Previous investigations devoted to the optimization of nonspherical gold nanoparticles for photothermal therapy (PTT) encountered two issues, namely, the appropriate selection of objective functions and the processing of particle random orientations. In this study, these issues were resolved, and accurate optimization results were obtained for the three typical nonspherical gold nanoparticles (nanospheroid, nanocylinder, and nanorod) by using the T-matrix method. The dependence of the optimization results on the excitation wavelength and the refractive index of tissue was investigated. Regardless of the excitation wavelength and tissue type, gold nanospheroids were found to be the most effective therapeutic agents for PTT. The light absorption ability of optimized nanoparticles could be enhanced by using a laser with a longer wavelength. Finally, the design tolerance for the different sizes of nanoparticles was provided.
\end{abstract}

Keywords: gold nanoparticles; light absorption; photothermal therapy; T-matrix method; optimization

\section{Introduction}

Gold nanoparticles can strongly absorb the incident light at the resonance wavelength. Almost all of the absorbed light is converted to heat via a series of nonradiative processes [1]. Moreover, gold nanoparticles are biocompatible and nontoxic. Therefore, they can easily penetrate a tumor upon systemic delivery and can be easily conjugated to cancer cells. Based on the above-mentioned properties, gold nanoparticles can be used as an effective therapeutic agent for the photothermal therapy (PTT) of cancer [1-4]. The enhancement of the light absorption ability of gold nanoparticles reduces the dosage of gold, shortens the illumination time, and decreases the damage of healthy cells. The light absorption ability of gold nanoparticles with a fixed shape is strongly dependent on the particle size [5-7]. Therefore, it is necessary to find the optimal size of gold nanoparticles by maximizing their light absorption ability at the excitation wavelength.

It is well known that experimental investigations on PTT are limited with respect to the prepared samples. Some of the samples can strongly absorb light at the target laser wavelength without ensuring their maximum light absorption ability. In order to find the optimal size of gold nanoparticles for 
PTT, some numerical optimizations were performed on gold nanoparticles [8-12]. However, to the best of our knowledge, we found two issues which led to inaccurate optimization results. Firstly, it is unreasonable to use the absorption efficiency $Q_{a b s}$ as an objective function in optimization. The $Q_{a b s}$ is defined as $C_{a b s} / S$, where $C_{a b s}$ is the absorption cross-section which represents the light absorption energy by a single particle, and $S$ is the particle cross-sectional area projected onto a plane perpendicular to the incident light [13]. Assuming that a fixed volume of bulk gold is used to prepare nanoparticles, the appropriate size of the nanoparticles which can most effectively absorb light of a given wavelength should then be selected. Clearly, $C_{a b s} / V$ ( $V$ is the particle volume) should be computed as a function of size rather than of $Q_{a b s}$ [14], in order to fulfill the above-mentioned criteria. Secondly, the random orientation of nonspherical particles, which is an important parameter affecting their light absorption abilities, is not considered in optimization. Kessentini and Barchiesi [10] considered the circular polarization of incident light to take into account the random orientation, but it was a conceptual mistake [13]. In this study, the above-mentioned two issues were solved and accurate optimal size parameters of three typical nonspherical gold nanoparticles (nanospheroid, nanocylinder, and nanorod) were obtained for PTT.

The remainder of this paper is organized as follows: Section 2 describes the calculation of light absorption by nonspherical gold nanoparticles. Section 3 presents the method for obtaining the optimal size of gold nanoparticles at typical laser wavelengths used in PTT. The effects of the laser wavelength and the refractive index of tissue on the optimization results are discussed. Moreover, the ranges of size parameters that keep the volume absorption coefficient greater than a threshold are computed. Finally, Section 4 concludes the paper.

\section{Light Absorption by Nonspherical Gold Nanoparticles}

The geometrical models of a gold nanospheroid, nanocylinder, and nanorod are shown in Figure 1. The gold nanorod is considered to have the geometry of a cylinder capped with two hemispheres. Two size parameters, length $L$ and diameter $D$, are used to describe all three types of shapes. The length $L$ and the aspect ratio $R=L / D$ are used for the optimization of these nanoparticles. Notably, a prolate spheroid and cylinder, with their length larger than their diameter $(L>D)$, are considered in this study.

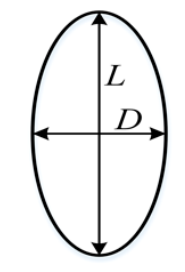

(a) nanospheroid

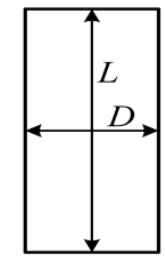

(b) nanocylinder

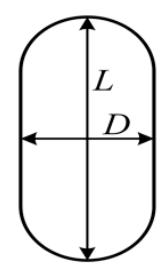

(c) nanorod

Figure 1. Geometrical models of gold (a) nanospheroid, (b) nanocylinder, and (c) nanorod. The $L$ represents length and the $D$ represents diameter.

All three types of nanoparticles belong to the class of rotationally symmetric particles. Moreover, the nanoparticles are randomly oriented in therapeutic applications. Fortunately, the light absorption properties of randomly oriented, rotationally symmetric metal nanoparticles can be easily simulated by the T-matrix method [15]. Furthermore, the absorption cross-section of homogeneous, randomly oriented, and rotationally symmetric particles is given by the Formula (1) [16] as follows:

$$
C_{a b s}=-\frac{2 \pi}{k^{2}} \operatorname{Re} \sum_{n=1}^{\infty} \sum_{m=-n}^{n}\left[T_{m n m n}^{11}+T_{m n m n}^{12}\right]-\frac{2 \pi}{k^{2}} \sum_{n=1}^{\infty} \sum_{n^{\prime}=1}^{\infty} \sum_{m=-n}^{n} \sum_{m^{\prime}=-n^{\prime}}^{n^{\prime}} \sum_{i=1}^{2} \sum_{j=1}^{2}\left|T_{m n m^{\prime} n^{\prime}}^{i j}\right|^{2},
$$

where $k$ is the wavenumber in the surrounding medium and $T$ is a matrix. The details of the T-matrix method can be found in a book written by Mishchenko et al. [16]. The absolute magnitude of the 
absorption cross-section does not provide a reliable measure for the light absorption of an ensemble of nanoparticles employed in real-life applications, because smaller particles can be loaded in a given volume in greater numbers compared to particles with a larger size. Therefore, the more meaningful parameter for comparison across a range of sizes includes the absorption cross-section per unit particle volume called volume absorption coefficient $\alpha_{a b s}$. Volume absorption coefficient $\alpha_{a b s}$ is calculated herein to compare the light absorption ability of the three types of nanoparticles. The $\alpha_{a b s}$ is given by Formula (2) as follows:

$$
\alpha_{a b s}=\frac{C_{a b s}}{V}
$$

where $V$ is the particle volume.

The refractive index of particles is an important parameter for the calculation. When the light interacts with the metal nanoparticles, especially for sizes smaller than the mean free path of the free electrons, the complex refractive index of the nanoparticle deviates from the bulk value because the collision of the free electrons with the particle surface becomes important and cannot be neglected [17]. The complex refractive index becomes size-dependent, and it can be expressed as [17]:

$$
n_{p}=\sqrt{n_{b u l k}^{2}(\omega)+\frac{\omega_{p}^{2}}{\omega^{2}+i \omega v_{f} / l_{\infty}}-\frac{\omega_{p}^{2}}{\omega^{2}+i \omega\left(v_{f} / l_{\infty}+A v_{f} / L_{e f f}\right)}},
$$

where $n_{\text {bulk }}$ is the complex refractive index of bulk metals, $\omega$ is the angle frequency of incident light, $\omega_{p}$ is the plasma frequency, $v_{f}$ denotes the Fermi velocity, $l_{\infty}$ represents the mean free path of the free electrons, $A$ is a dimensionless parameter, usually assumed to be equal to 1 , and $L_{\text {eff }}$ is the reduced effective mean free path of the free electrons which equals the particle effective radius (equal-volume-sphere radius) [17]. For gold, $\hbar \omega_{p}=9.03 \mathrm{eV}$, which is obtained from the literature report [18], $v_{f}=1.40 \times 10^{15} \mathrm{~nm} / \mathrm{s}$ and $l_{\infty}=42 \mathrm{~nm}$, which are acquired from the literature study [19]. The values of $n_{\text {bulk }}$ at different wavelengths are obtained from the data published by Johnson and Christy [20]. Cubic spline interpolation was used to calculate the complex refractive indices at intermediate wavelengths, when the data are not available directly from Johnson and Christy.

The computer codes for the calculation of $\alpha_{a b s}$ were developed based on the free T-matrix codes developed by Mishchenko (https://www.giss.nasa.gov/staff/mmishchenko/t_matrix.html) [21]. Furthermore, the shape of the nanorods and the size-dependent complex refractive index of the metal nanoparticles were added to the source code. The main input parameters for the code included the light wavelength $\lambda$, the aspect ratio $R$, the length $L$ of particles, and the refractive indices of particles and the surrounding medium, $n_{p}$ and $n_{m}$, respectively. In most calculations, the refractive index of the surrounding tissue was taken as 1.44 , which is the refractive index of the subcutaneous fat [22]. Nanoparticles with length within the range of 30-130 nm were considered, and the aspect ratio was selected between 2 and 8 . The step sizes of the length and the aspect ratio were considered to be $1 \mathrm{~nm}$ and 0.1 , respectively.

\section{Results and Discussion}

To obtain the optimal size parameters, the values of the volume absorption coefficient $\alpha_{a b s}$ of the gold nanospheroid, nanocylinder, and nanorod were calculated at an excitation wavelength of $800 \mathrm{~nm}$ as a function of the aspect ratio and length (Figure 2). Clearly, all three particles exhibited a distinct peak corresponding to the maximum $\alpha_{a b s}$. Data obtained in this study indicate that the optimized nanospheroids with an aspect ratio of 3.7 and a length of $59 \mathrm{~nm}$ exhibited the best light absorption ability compared to that of the optimized nanocylinders and nanorods. The maximum $\alpha_{a b s}$ of a nanospheroid is $8.7 \%$ higher than that of a nanocylinder, and $9.7 \%$ higher than that of a nanorod. Kessentini and Barchiesi [10] reported that the absorption efficiency of optimized nanorods was slightly higher than those of optimized nanospheroids and nanocylinders. Their results were inconsistent 
with the results of this study because of the different selections of objective function and processing of particle random orientation.
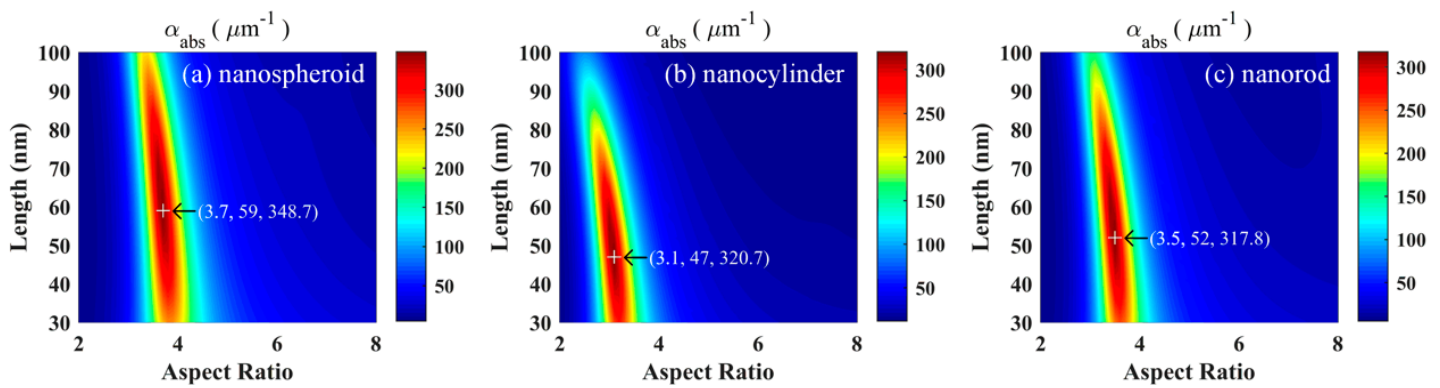

Figure 2. Volume absorption coefficient $\alpha_{a b s}$ of gold (a) nanospheroid, (b) nanocylinder, and (c) nanorod at an excitation wavelength of $800 \mathrm{~nm}$ as a function of the aspect ratio and length. The plus sign (+) is a point corresponding to the maximum $\alpha_{a b s}$, and the numbers in the brackets are the values of optimal aspect ratio, optimal length, and maximum $\alpha_{a b s}$, respectively. The refractive index of tissue is 1.44.

To provide more comprehensive data for experimental workers, the optimized size parameters and the corresponding maximum volume absorption coefficients of gold nanospheroids, nanocylinders, and nanorods were computed at four typical excitation wavelengths $(800,808,820$, and $1064 \mathrm{~nm})$ commonly used in PTT (Table 1). The results indicated that the light absorption ability of optimized nanoparticles could be enhanced by using lasers with longer wavelengths. The volume absorption coefficient of an optimized nanospheroid at a wavelength of $1064 \mathrm{~nm}$ was found to be $26.8 \%$ higher than that at $800 \mathrm{~nm}$. The optimal size parameters of the gold nanoparticles became larger with the increase of the excitation wavelength. Gold nanoshells are the most commonly used nanoparticles in PTT. The light absorption ability of a hollow gold nanoshell, calculated by using the Mie theory of coated sphere $[13,14]$, was compared with that of a gold nanospheroid (Table 2). The maximum volume absorption coefficient of a gold nanospheroid was found to be three times that of a gold nanoshell at a wavelength of $800 \mathrm{~nm}$. With an increase in the wavelength, the gap between the two nanoparticles became larger. In short, the optimized nanospheroids exhibited the best light absorption ability at all excitation wavelengths.

Table 1. Maximum volume absorption coefficients $\alpha_{a b s, \max }$ and the corresponding optimal aspect ratio $R_{\text {opt }}$ and optimal length $L_{\text {opt }}$ of gold nanospheroid, nanocylinder, and nanorod at four typical excitation wavelengths $(800,808,820$, and $1064 \mathrm{~nm})$. The refractive index of tissue is 1.44 .

\begin{tabular}{ccccc}
\hline$\lambda(\mathbf{n m})$ & Shape & $\boldsymbol{R}_{\text {opt }}$ & $\boldsymbol{L}_{\text {opt }}(\mathbf{n m})$ & $\boldsymbol{\alpha}_{\boldsymbol{a b s , \boldsymbol { m a x }}}\left(\boldsymbol{\mu \mathbf { m } ^ { - 1 } )}\right.$ \\
\hline \multirow{3}{*}{800} & nanospheroid & 3.7 & 59 & 348.7 \\
& nanocylinder & 3.1 & 47 & 320.7 \\
& nanorod & 3.5 & 52 & 317.8 \\
\hline \multirow{3}{*}{808} & nanospheroid & 3.7 & 66 & 352.1 \\
& nanocylinder & 3.1 & 52 & 324.9 \\
& nanorod & 3.5 & 58 & 322.9 \\
\hline \multirow{3}{*}{820} & nanospheroid & 3.8 & 68 & 359.0 \\
& nanocylinder & 3.2 & 53 & 332.2 \\
& nanorod & 3.6 & 59 & 330.0 \\
\hline \multirow{3}{*}{1064} & nanospheroid & 5.9 & 94 & 442.0 \\
& nanocylinder & 5.1 & 74 & 409.5 \\
& nanorod & 5.4 & 84 & 407.5 \\
\hline
\end{tabular}


Table 2. Maximum volume absorption coefficients $\alpha_{a b s, \max }$ of gold nanospheroids and nanoshells (hollow core) at four typical excitation wavelengths $(800,808,820$, and $1064 \mathrm{~nm})$. The refractive index of tissue is 1.44 .

\begin{tabular}{cccc}
\hline \multirow{2}{*}{$\lambda(\mathrm{nm})$} & \multicolumn{2}{c}{$\alpha_{a b s, \max }\left(\mu \mathrm{m}^{-\mathbf{1}}\right)$} & $\alpha_{a b s, \max }^{\text {nanospheroid }} / \alpha_{a b s, \max }^{\text {nanoshell }}$ \\
\cline { 2 - 3 } & Nanospheroid & Hollow Nanoshell & 3.0 \\
\hline 800 & 348.7 & 117.4 & 3.1 \\
808 & 352.1 & 114.9 & 3.2 \\
820 & 359.0 & 111.4 & 7.1 \\
1064 & 442.0 & 62.5 & \\
\hline
\end{tabular}

To investigate the effect of the excitation wavelength on the optimization results, wavelengths from 800 to $1100 \mathrm{~nm}$ were selected because they are the main wavelength range for lasers used in PTT. The optimization results for the three types of gold nanoparticles at the wavelength range between 800 and $1100 \mathrm{~nm}$ are shown in Figure 3. Due to the increase of the excitation wavelength, the light absorption ability of each nanoparticle becomes higher (Figure 3a), the optimal aspect ratios of nanoparticles increase almost linearly (Figure $3 b$ ), and the optimal lengths of nanoparticles increase in a stepwise manner (Figure 3c). The optimized nanospheroids exhibited the highest light absorption ability at all wavelengths, while the optimized nanocylinders showed a slightly higher light absorption ability than that of the optimized nanorods. Both the optimal aspect ratio and the length of a nanospheroid were larger than those of a nanocylinder and nanorod, while the optimized nanocylinder displayed smaller size parameters than that of the optimized nanorod. Due to the increase in the wavelength from 800 to $1100 \mathrm{~nm}$, the maximum volume absorption coefficient of the nanospheroid increased from 348.7 to $450.3 \mathrm{\mu m}^{-1}$, the optimal aspect ratio of the nanospheroid increased from 3.7 to 6.2 , and its optimal length exhibited an increase from 59 to $100 \mathrm{~nm}$.
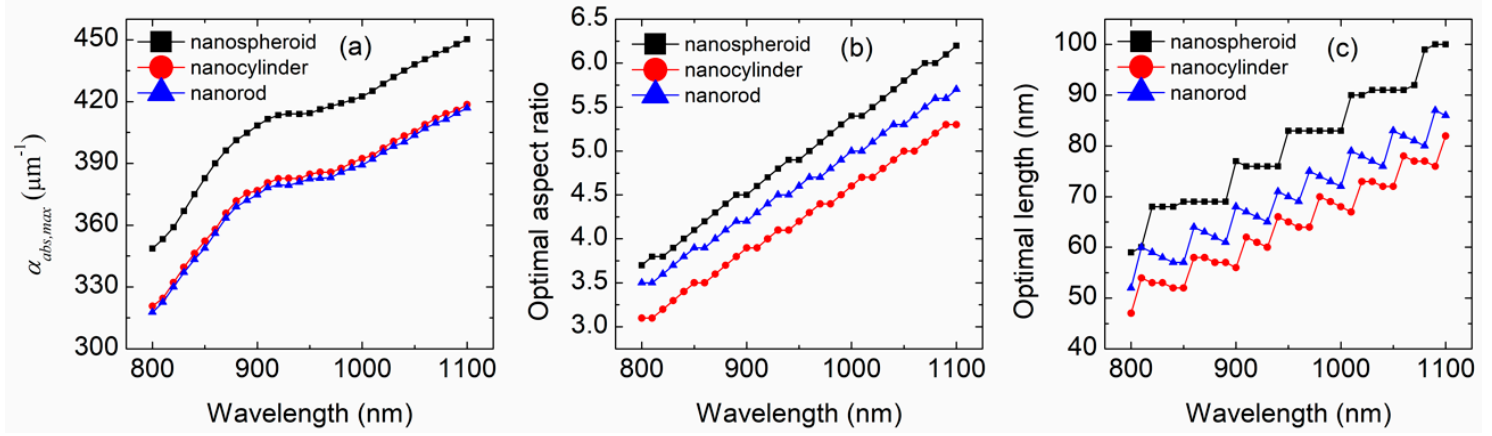

Figure 3. (a) Maximum volume absorption coefficient $\alpha_{a b s, m a x}$, (b) optimal aspect ratio $R_{\text {opt }}$, and (c) optimal length $L_{\text {opt }}$ of gold nanospheroid, nanocylinder, and nanorod at excitation wavelengths from 800 to $1100 \mathrm{~nm}$ at a step size of $10 \mathrm{~nm}$. The refractive index of tissue is 1.44 .

The refractive index of the surrounding medium is an important parameter impacting the light absorption property of gold nanoparticles. In the calculations performed in this study, the surrounding medium was considered to be biological tissue with a refractive index between 1.35 and 1.7 [22]. Figure 4 demonstrates the effect of the refractive index of tissue on the optimization results. Due to the increase of the refractive index of tissue, the light absorption ability of each nanoparticle decreases almost linearly (Figure 4a), and the optimal size parameters of the nanoparticles become smaller (Figure $4 \mathrm{~b}, \mathrm{c}$ ). Clearly, the change of the refractive index of tissue does not make the light absorption ability of a nanospheroid lose its dominant position. Both the optimal aspect ratio and the length of a nanocylinder are smaller than those of a nanospheroid and a nanorod. An optimized nanospheroid has larger size parameters than an optimized nanorod. With the increase in the refractive index from 1.35 to 1.7, the maximum volume absorption coefficient of a nanospheroid decreases from 369.8 to 
$305.2 \mu \mathrm{m}^{-1}$, the optimal aspect ratio of a nanospheroid decreases from 4 to 2.9 , and its optimal length decreases from 69 to $49 \mathrm{~nm}$.
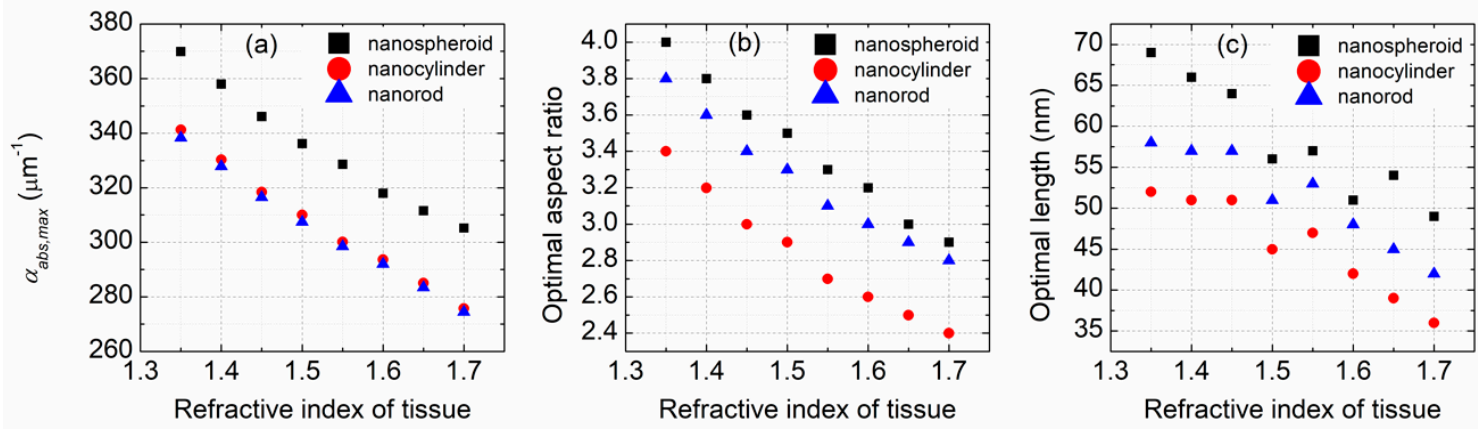

Figure 4. (a) Maximum volume absorption coefficients $\alpha_{a b s, \max },(\mathbf{b})$ optimal aspect ratio $R_{\text {opt }}$, and (c) optimal length $L_{\text {opt }}$ of gold nanospheroid, nanocylinder, and nanorod at an excitation wavelength of $800 \mathrm{~nm}$. The refractive index of tissue is varied from 1.35 to 1.7 in a step size of 0.05 .

The above-mentioned discussion clearly indicates the presence of only one group of optimized size parameters for a fixed excitation wavelength and a known biological tissue. However, it is difficult to prepare the gold nanoparticles of the same size in the actual particle synthesis as the optimized results. Therefore, it is necessary to obtain the ranges of size parameters that keep the volume absorption coefficient greater than a threshold. Herein, a threshold of $90 \%$ of the maximum volume absorption coefficient was considered for each nanoparticle. Figure 5 shows the size parameters of gold nanoparticles, ensuring that the volume absorption coefficients are larger than $90 \%$ of the maximum coefficients, at an excitation wavelength of $800 \mathrm{~nm}$. The range of length is much wider than that of the aspect ratio for each nanoparticle. Compared to the nanocylinder and nanorod, the nanospheroid exhibited a slightly wider range of length and a larger aspect ratio. The min-max and mean-std values of the aspect ratio and length of the gold nanoparticles, ensuring that the volume absorption coefficients are larger than $90 \%$ of the maximum coefficients, at four typical excitation wavelengths $(800,808,820$, and $1064 \mathrm{~nm})$, are listed in Table 3. The results indicate that the nanoparticles have larger size parameters and wider ranges at the longer excitation wavelength.
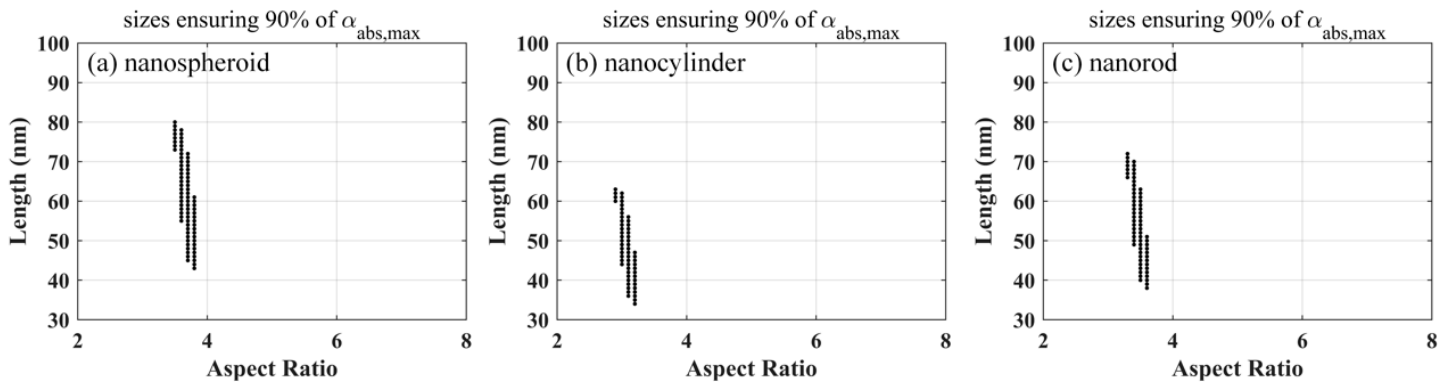

Figure 5. Aspect ratio and length of the gold (a) nanospheroid, (b) nanocylinder, and (c) nanorod at an excitation wavelength of $800 \mathrm{~nm}$, ensuring that the volume absorption coefficients are larger than $90 \%$ of the maximum coefficients. The refractive index of tissue is 1.44 . 
Table 3. Min, max, mean, and std values of aspect ratio $R$ and length $L$, ensuring that the volume absorption coefficients are larger than $90 \%$ of the maximum coefficients, at four typical excitation wavelengths $(800,808,820$, and $1064 \mathrm{~nm})$. The refractive index of tissue is 1.44 .

\begin{tabular}{cccccc}
\hline$\lambda(\mathbf{n m})$ & Shape & {$\left[\boldsymbol{R}_{\text {min }}, \boldsymbol{R}_{\text {max }}\right]$} & $\boldsymbol{R}_{\text {mean }} \pm \sigma_{\boldsymbol{R}}$ & {$\left[\boldsymbol{L}_{\text {min }}, \boldsymbol{L}_{\text {max }}\right](\mathbf{n m})$} & $\boldsymbol{L}_{\text {mean }} \pm \sigma_{L}(\mathbf{n m})$ \\
\hline \multirow{3}{*}{800} & nanospheroid & {$[3.5,3.8]$} & $3.7 \pm 0.1$ & {$[43,80]$} & $61 \pm 10$ \\
& nanocylinder & {$[2.9,3.2]$} & $3.1 \pm 0.1$ & {$[34,63]$} & $48 \pm 8$ \\
& nanorod & {$[3.3,3.6]$} & $3.5 \pm 0.1$ & {$[38,72]$} & $54 \pm 9$ \\
\hline \multirow{2}{*}{808} & nanospheroid & {$[3.6,3.9]$} & $3.7 \pm 0.1$ & {$[44,82]$} & $62 \pm 10$ \\
& nanocylinder & {$[3.0,3.3]$} & $3.1 \pm 0.1$ & {$[35,65]$} & $50 \pm 8$ \\
& nanorod & {$[3.4,3.6]$} & $3.5 \pm 0.1$ & {$[39,73]$} & $57 \pm 9$ \\
\hline \multirow{2}{*}{820} & nanospheroid & {$[3.7,4.0]$} & $3.9 \pm 0.1$ & {$[45,84]$} & $64 \pm 10$ \\
& nanocylinder & {$[3.1,3.4]$} & $3.2 \pm 0.1$ & {$[36,66]$} & $51 \pm 8$ \\
& nanorod & {$[3.5,3.7]$} & $3.6 \pm 0.1$ & {$[40,74]$} & $57 \pm 9$ \\
\hline \multirow{2}{*}{1064} & nanospheroid & {$[5.6,6.2]$} & $5.9 \pm 0.2$ & {$[64,122]$} & $93 \pm 15$ \\
& nanocylinder & {$[4.8,5.3]$} & $5.1 \pm 0.2$ & {$[52,99]$} & $75 \pm 12$ \\
& nanorod & {$[5.2,5.7]$} & $5.5 \pm 0.2$ & {$[56,106]$} & $80 \pm 13$ \\
\hline
\end{tabular}

\section{Conclusions}

In this study, the light absorption ability of a gold nanospheroid, nanocylinder, and nanorod was investigated in the near-infrared region. The optimal size parameters of nanoparticles were obtained by maximizing the volume absorption coefficient. Four typical excitation wavelengths $(800,808,820$, and $1064 \mathrm{~nm}$ ) used in PTT were considered. The results showed that the optimized nanospheroids with an aspect ratio of 5.9 and a length of $94 \mathrm{~nm}$ exhibited the best light absorption ability at the excitation wavelength of $1064 \mathrm{~nm}$. In this case, the light absorption ability of optimized nanospheroids was found to be 7.1 times that of optimized nanoshells. Furthermore, the effects of the excitation wavelength and the refractive index of tissue on the optimization results were analyzed. Both the light absorption ability and the size parameters of optimized nanoparticles increased with the increase of the excitation wavelength, while they decreased as the refractive index of tissue increased. Finally, the ranges of the size parameters that maintained the volume absorption coefficient greater than a threshold of $90 \%$ of maximum value were calculated. The optimized particles are suitable for continuous wave and pulsed laser illumination because the T-matrix method is independent of the laser output mode. This study offers a purely theoretical treatment of the optimization of gold nanoparticle manufacture for PTT. Undeniably, more systematic explorations are demanded to investigate factors such as particle toxicity, melting threshold, and cellular uptake, which play important roles in the efficiency of PTT techniques.

Author Contributions: Conceptualization, P.T., X.H. and Y.Y.; methodology, P.T. and X.Y.; software, P.T. and X.Y.; validation, P.T., X.H. and Y.Y.; formal analysis, P.T. and X.H.; investigation, P.T.; resources, X.H.; data curation, P.T.; writing-original draft preparation, P.T. and X.Y.; writing-review and editing, P.T., X.H. and Y.Y.; visualization, P.T.; supervision, X.H. and Y.Y.; project administration, P.T.; funding acquisition, P.T.

Funding: This work was supported by the National Natural Science Foundation of China (Grant No. 11764042), the University Scientific Research Program of Xinjiang Uygur Autonomous Region (Grant No. XJEDU2016I032), the Hundred Young Doctor Introduction Plan of Xinjiang Uygur Autonomous Region, and the Foundation of Xinjiang Normal University Laboratory of Novel Light Source and Micro/Nano-Optics (Grant No. XJNUSYS092017A02).

Acknowledgments: We express our gratitude to M. I. Mishchenko (NASA Goddard Institute for Space Studies) for use of the free T-matrix code.

Conflicts of Interest: The authors declare no conflict of interest. The funders had no role in the design of the study; in the collection, analyses, or interpretation of data; in the writing of the manuscript, or in the decision to publish the results. 


\section{References}

1. Huang, X.; El-Sayed, M.A. Gold nanoparticles: Optical properties and implementations in cancer diagnosis and photothermal therapy. J. Adv. Res. 2010, 1, 13-28. [CrossRef]

2. Huang, X.; Jain, P.K.; El-Sayed, I.H.; El-Sayed, M.A. Plasmonic photothermal therapy (PPTT) using gold nanoparticles. Lasers Med. Sci. 2008, 23, 217-228. [CrossRef] [PubMed]

3. Ali, M.R.K.; Farghali, H.A.M.; Wu, Y.; El-Sayed, I.; Osman, A.H.; Selim, S.A.; El-Sayed, M.A. Gold nanorod-assisted photothermal therapy decreases bleeding during breast cancer surgery in dogs and cats. Cancers 2019, 11, 851. [CrossRef] [PubMed]

4. Vines, J.B.; Yoon, J.; Ryu, N.; Lim, D.; Park, H. Gold nanoparticles for photothermal cancer therapy. Front. Chem. 2019, 7, 167. [CrossRef] [PubMed]

5. Link, S.; El-Sayed, M.A. Size and temperature dependence of the plasmon absorption of colloidal gold nanoparticles. J. Phys. Chem. B 1999, 103, 4212-4217. [CrossRef]

6. Jain, P.K.; Lee, K.S.; El-Sayed, I.H.; El-Sayed, M.A. Calculated absorption and scattering properties of gold nanoparticles of different size, shape, and composition: Applications in biological imaging and biomedicine. J. Phys. Chem. B 2006, 110, 7238-7248. [CrossRef] [PubMed]

7. Tuersun, P.; Han, X. Optical absorption analysis and optimization of gold nanoshells. Appl. Opt. 2013, 52, 1325-1329. [CrossRef] [PubMed]

8. Grosges, T.; Barchiesi, D.; Toury, T.; Gréhan, G. Design of nanostructures for imaging and biomedical applications by plasmonic optimization. Opt. Lett. 2008, 33, 2812-2814. [CrossRef] [PubMed]

9. Grosges, T.; Barchiesi, D.; Kessentini, S.; Gréhan, G.; de la Chapelle, M.L. Nanoshells for photothermal therapy: A Monte-Carlo based numerical study of their design tolerance. Biomed. Opt. Express 2011, 2, 1584-1596. [CrossRef] [PubMed]

10. Kessentini, S.; Barchiesi, D. Quantitative comparison of optimized nanorods, nanoshells and hollow nanospheres for photothermal therapy. Biomed. Opt. Express 2012, 3, 590-604. [CrossRef] [PubMed]

11. Sikdar, D.; Rukhlenko, I.D.; Cheng, W.; Premaratne, M. Effect of number density on optimal design of gold nanoshells for plasmonic photothermal therapy. Biomed. Opt. Express 2013, 4, 15-31. [CrossRef] [PubMed]

12. Sikdar, D.; Rukhlenko, I.D.; Cheng, W.; Premaratne, M. Optimized gold nanoshell ensembles for biomedical applications. Nanoscale Res. Lett. 2013, 8, 142. [CrossRef] [PubMed]

13. Bohren, C.F.; Huffman, D.R. Absorption and Scattering of Light by Small Particles; Wiley: New York, NY, USA, 1983.

14. Tuersun, P.; Han, X. Optimal dimensions of gold nanoshells for light backscattering and absorption based application. J. Quant. Spectrosc. Radiat. Transf. 2014, 146, 468-474. [CrossRef]

15. Khlebtsov, N.G. T-matrix method in plasmonics: An overview. J. Quant. Spectrosc. Radiat. Transf. 2013, 123, 184-217. [CrossRef]

16. Mishchenko, M.I.; Travis, L.D.; Lacis, A.A. Scattering, Absorption, and Emission of Light by Small Particles; Cambridge University Press: Cambridge, UK, 2002.

17. Keribig, U.; Vollmer, M. Optical Properties of Metal Clusters; Springer: Berlin, Germany, 1995.

18. Rakic, A.D.; Djurisic, A.B.; Elazar, J.M.; Majewski, M.L. Optical properties of metallic films for vertical-cavity optoelectronic devices. Appl. Opt. 1998, 37, 527-5283. [CrossRef] [PubMed]

19. Averitt, R.D.; Westcott, S.L.; Halas, N.J. Linear optical properties of gold nanoshells. J. Opt. Soc. Am. B 1999, 16, 1824-1832. [CrossRef]

20. Johnson, P.B.; Christy, R.W. Optical constants of the noble metals. Phys. Rew. B 1972, 6, 4370-4379. [CrossRef]

21. Mishchenko, M.I.; Travis, L.D. Capabilities and limitations of a current FORTRAN implementation of the T-matrix method for randomly oriented, rotationally symmetric scatterers. J. Quant. Spectrosc. Radiat. Transf. 1998, 60, 309-324. [CrossRef]

22. Duck, F.A. Physical Properties of Tissue: A Comprehensive Reference Book; Academic: London, UK, 1990.

(C) 2019 by the authors. Licensee MDPI, Basel, Switzerland. This article is an open access article distributed under the terms and conditions of the Creative Commons Attribution (CC BY) license (http://creativecommons.org/licenses/by/4.0/). 